\section{Consumo de micronutrientes e excesso de peso: existe relação?}

\section{Micronutrient consumption and overweight: Is there a relationship?}

\section{Ana Luisa Marcucci Leão' Luana Caroline dos Santos"}

'Grupo de Pesquisa em Intervenções em Nutrição da Universidade Federal de Minas Gerais - GIN-UFMG.

"Curso de Nutrição do Departamento de Enfermagem Materno-Infantil e Saúde Pública da Universidade Federal de Minas Gerais - UFMG, GIN-UFMG.

Correspondência: Luana Caroline dos Santos. Departamento de Enfermagem Materno-Infanti e Saúde Pública, Escola de Enfermagem, Universidade Federal de Minas Gerais. Avenida Professor Alfredo Balena, n 190, 4 andar, Sala 420. Belo Horizonte, MG CEP 30130-100. Email: luanacs@ig.com.br

\section{Resumo}

Introdução: O incremento da obesidade nas últimas décadas se associa às mudanças nos modos de vida da população, incluindo amplas alterações dietéticas, sobretudo na ingestão de micronutrientes. Objetivo: Descrever o consumo de micronutrientes e revisar sua implicação no estado nutricional. Métodos: Realizou-se levantamento bibliográfico contemplando artigos nacionais e internacionais, publicados nos últimos 11 anos, nas bases de dados SciELO, LILACS, Medline e sites de organizações governamentais, usando como descritores: "micronutrientes", "consumo alimentar", "excesso de peso" , "saúde" e "estado nutricional” e suas traduções em inglês. Resultados: A deficiência no consumo de micronutrientes é um problema de saúde global, atingindo cerca de 2 bilhões de pessoas, e parece se associar com maior risco de doenças e agravos não transmissíveis, incluindo a obesidade. A insuficiente ingestão de vitamina A favorece o excesso de peso por alteração no metabolismo da tireóide, enquanto a vitamina C se relaciona à síntese de carnitina e oxidação da gordura; e a vitamina D favorece o controle da saciedade e do gasto energético. Quanto aos minerais, o cálcio auxilia a regulação da termogênese e lipogênese/lipólise; e o zinco está envolvido na regulação de insulina e leptina. Conclusão: A promoção de hábitos alimentares saudáveis por meio de práticas educativas se faz necessária para maior conscientização da população acerca das implicações do consumo de micronutrientes no estado nutricional, devendo compor as estratégias de Saúde Pública de controle da obesidade e suas complicações.

Palavras-chave: Micronutrientes. Consumo de alimentos. Sobrepeso. Obesidade. Saúde. Estado nutricional. 


\section{Abstract}

Introduction: In past decades, the growth in obesity has been associated with changes in the lifestyle of the population, including comprehensive dietary changes, especially in the intake of micronutrients. Objective: To describe micronutrient intake and review its consequences on nutritional status. Methods: A literature review was performed covering domestic and international articles published over the past 11 years, on the SciELO, LILACS, MEDLINE and governmental databases, using descriptors such as "micronutrients", "food consumption", "excess weight", "health", "nutritional status" in Portuguese and in English. Results: Micronutrient intake deficiency is a global health problem, affecting about 2 billion people and seems to be associated with an increased risk for non-communicable diseases and disorders, including obesity. Insufficient intake of vitamin A favors overweight by altering thyroid metabolism, while vitamin $C$ is related to the synthesis of carnitine and fat oxidation; vitamin D favors the control of satiety and energy expenditure. As for minerals, calcium helps the regulation of thermogenesis and lipogenesis / lipolysis, and zinc is involved in regulating insulin and leptin. Conclusion: The promotion of healthy eating habits through educational practices is necessary for greater public awareness of the implications of micronutrient intake on nutritional status, and should be included in Public Health strategies in order to control obesity and its complications.

Keywords: Micronutrient. Food consumption. Overweight. Obesity. Health. Nutritional status.

\section{Introdução}

A obesidade é definida como o acúmulo excessivo de tecido adiposo no organismo, de forma a comprometer a saúde dos indivíduos. É considerada fator de risco para enfermidades, tais como dislipidemias, diabetes mellitus tipo II, doenças cardiovasculares e alguns tipos de câncer ${ }^{1}$. Possui etiologia complexa, apresentando caráter multifatorial e contemplando aspectos culturais, ambientais, genéticos, socioeconômicos, biológicos e psicossociais ${ }^{2}$.

Considera-se a obesidade como um problema de saúde pública, tanto nos países em desenvolvimento quanto nos desenvolvidos, denotando-se uma epidemia mundial. De acordo com dados da Organização Mundial de Saúde (OMS) esse agravo nutricional atinge possivelmente $10 \%$ da população adulta ${ }^{1}$. E, em 2025 , estima-se ocorrência de 300 milhões de obesos no mundo ${ }^{3}$.

No Brasil, a análise de cinco estudos de base populacional realizados no país - Estudo Nacional sobre Despesas Familiares (ENDEF), realizado entre 19741975; a Pesquisa Nacional sobre Saúde e Nutrição (PNSN), de 1989; a Pesquisa sobre Padrões de Vida (PPV), desenvolvida em 1996-1997'; e a Pesquisa de Orçamentos Familiares $^{4}$ (POF), de 2002-2003 e 2008$2009^{4}$, permitiu avaliar a magnitude dos agravos nutricionais mais relevantes na população, incluindo a emergência da obesidade, bem como verificar seus principais determinantes. De acordo com esses estudos, a prevalência da desnutrição em crianças e adultos declinou nas últimas décadas, enquanto o sobrepeso e a obesidade aumentaram na população brasileira, principalmente entre os adultos.

Nota-se que a prevalência de excesso de peso em adultos aumentou em quase três vezes no sexo masculino (de 18,5\% para $50,1 \%$ ) e em quase duas vezes no sexo feminino (de $28,7 \%$ para $48,0 \%)^{4}$. No mesmo período (1974-2009), a prevalência de obesidade aumentou em mais de quatro vezes para homens (de 2,8\% para 12,4\%) e 
em mais de duas vezes para mulheres (de $8,0 \%$ para $16,9 \%)^{5}$.

A ascensão da obesidade se associa às mudanças ocorridas nos padrões de atividade física e alimentação das populações ${ }^{1,6} \mathrm{em}$ virtude de mudanças econômicas, sociais e demográficas decorrentes do processo de modernização mundial ${ }^{1,6}$.

$\mathrm{Na}$ alimentação, verificou-se aumento da quantidade calórica que pode ser consequência da elevada quantidade do consumo de alimentos ou de mudanças qualitativas na dieta, no que concerne ao maior consumo de alimentos com alta densidade energética ${ }^{7}$.

Os dados do Instituto Brasileiro de Geografia e Estatística (IBGE) ${ }^{9}$ demonstram que entre o período de 1974 a 2003 houve redução de $46 \%$ na aquisição domiciliar de arroz polido, $37 \%$ de feijão, $10 \%$ de carne bovina e $41 \%$ de frango, e aumento de $216 \%$ na aquisição de alimentos preparados. Ademais, a insuficiência no consumo de frutas, legumes e verduras na dieta dos brasileiros foi constatada em todos os segmentos populacionais ${ }^{8} \mathrm{e}$ foi confirmada em estudos posteriores ${ }^{6}$. Em consequência, a deficiência de micronutrientes aparece como um problema de saúde global, atingindo cerca de 2 bilhões de pessoas no mundo 9 .

No Brasil existe uma grande limitação sobre o consumo de micronutrientes, porém os achados do estudo Brazos $^{10}$, com 2.420 participantes em 150 municípios das cinco regiões do País, concluiu que a ingestão de vitaminas e minerais é inadequada na dieta padrão do país, independente da classe social. A inadequação no consumo de vitaminas e minerais foi de $50 \%$ para vitamina $A, 80 \%$ para vitamina $C$ e para magnésio, $81 \%$ para vitamina Ke, $99 \%$ para vitaminas E e D.

Estudos internacionais também revelam inadequada ingestão de micronutrientes. Dois estudos transversais consecutivos, ENCAT 1992-93 e 2002-03 ${ }^{11}$, realizados na população catalã com 4.071 participantes, investigaram a ingestão dietética por meio de dois recordatórios 24 horas em dias não consecutivos e constataram redução acentuada na ingestão de vitamina A (de 796 mg para 429, $\mathrm{p}<0,001)$ e vitamina $\mathrm{D}(-20,2$ mg para homens e-20,9 mg para mulheres); e aumento da ingestão de vitamina C (+ $117,6 \mathrm{mg}$ por dia).

O consumo insuficiente de micronutrientes está entre os dez principais fatores de risco para a carga total global de doenças em todo o mundo, sendo considerado o terceiro fator de risco prevenível de doenças e agravos não-transmissíveis (DANT) ${ }^{12}$. A associação com a adiposidade também parece existir. Azadbakht et al. ${ }^{13}$ avaliaram 926 mulheres residentes no distrito de Teerã, no Irã, e identificaram significativa associação entre adiposidade central e baixa ingestão de vitamina C (odds ratio: 2,31; IC95\% 1,25-4,25) e de cálcio (odds ratio: 1,30; IC $95 \% 1,07-3,78)^{13}$.

Nesse cenário, a avaliação do consumo alimentar mostra-se essencial como fator de compreensão da relação entre alimentação e saúde, sobretudo no que se refere à ingestão de alguns micronutrientes e estado nutricional. Adicionalmente, considerando que o consumo adequado de micronutrientes é essencial para manutenção de diversas funções metabólicas do organismo, o objetivo do presente trabalho foi investigar o consumo de micronutrientes na população e sua possível implicação no estado nutricional.

\section{Métodos}

Trata-se de um estudo de revisão, pautado em artigos nacionais e internacionais, publicados nos últimos 11 anos (2000-2011), que abrangeram investigações de micronutrientes e suas possíveis implicações no estado nutricional. As bases de dados consultadas foram: Scientific Eletronic Library Online (SciELO), Literatura Latino-Americana e do Caribe em Ciências da Saúde (LILACS) e Medical Literature Analysis and Retrieval System Online (Medline) da National Library of Medicine. Adicionalmente, realizou-se busca da temática em estudo em sites de instituições e organizações governamentais, tais como Organização Mundial de Saúde e Ministério da Saúde. Foram utilizados os 
descritores: "consumo de micronutrientes", "consumo alimentar", "excesso de peso", "estado nutricional" e "saúde", e suas respectivas traduções para a língua inglesa.

\section{Resultados}

\section{Consumo de micronutrientes na população brasileira e mundial}

No Brasil, poucos estudos contemplaram a avaliação do consumo de micronutrientes na população. Estudo realizado, em 150 municípios brasileiros, identificou ampla inadequação no consumo de micronutrientes, tais como vitaminas A (50\%), C $(80 \%)$, E e D $(99 \%)^{10}$.

Outro estudo, realizado na cidade de Bambuí-MG ${ }^{14}$, avaliou o consumo alimentar de 550 indivíduos adultos e idosos por meio do Questionário Semiquantitativo de Frequência Alimentar e Recordatório Alimentar de 24 horas, e verificou baixo percentual da população com razão de adequação de nutrientes recomendada para o consumo de vitaminas $(0,0-5,1 \%)$ e minerais $(0-21,1 \%)$. Notificou-se inadequação similar entre os sexos para zinco e vitamina A $(99,8 \%)$, cálcio (98\%) e vitamina C (93,8\%).

Meléndez et al. ${ }^{15}$ verificaram diferenças no consumo de vitaminas e minerais em relação ao sexo e idade na população residente em área metropolitana de São Paulo. O total de indivíduos estudados foi de 548, com idades compreendidas entre 20 e 88 anos. Constatou-se maior consumo de algumas vitaminas e minerais em indivíduos do sexo masculino e nos grupos etários de indivíduos mais jovens. De grande importância do ponto de vista da saúde pública foram os valores medianos de consumo de vitamina A (496 $\mu \mathrm{g} /$ dia) e cálcio (379-432 mg entre os homens; $240-378 \mathrm{mg}$ entre as mulheres). Os idosos apresentaram níveis de ingestão menores do que os mais jovens ( $p<0,00001$ ), mas destaca-se que adultos e idosos não atingiram as recomendações de consumo para populações da América Latina, sendo a de vitamina A de 915 e $660 \mu \mathrm{g} /$ dia para ambos, respectivamente, e a de cálcio de 925 a 1,525 mg/dia. Tais achados indicaram risco de deficiência nessa população.

Corroborando estes achados, Abreu et al. ${ }^{16}$ avaliaram o consumo alimentar de idosos residentes na cidade de Viçosa/ MG por meio do recordatório 24 horas e questionário de frequência alimentar semiquantitativo (QFA), e constataram elevada inadequação no consumo de vitaminas $\mathrm{C}$ $(40,5 \%)$ e A $(70,8 \%)$, enquanto o consumo médio de cálcio foi cerca de $1 / 3$ do valor recomendado.

Assim como na população brasileira, dados de estudos mundiais revelam consumo insuficiente de micronutrientes em diferentes cenários de investigação e apontam possíveis diferenças segundo o estado nutricional. Suliburska et al. ${ }^{17}$ analisaram o consumo alimentar de 40 indivíduos obesos hipertensos, com resistência à insulina - grupo caso e 40 indivíduos saudáveis - grupo controle. Identificou-se baixo consumo de zinco $(10,3 \pm 1,1$ vs 14,3 $\pm 2,1 \mathrm{mg})$ e cálcio, $(507,3 \pm 59,1$ vs $842,4 \pm$ $65,6 \mathrm{mg})$, principalmente entre aqueles com obesidade, $\mathrm{p}<0,005$.

De modo similar, estudo transversal com 3.421 adultos do sul da Espanha ${ }^{18}$ detectou elevada prevalência de inadequação no consumo de zinco $(56,45 \%)$ e apontou menores níveis, dietéticos e plasmáticos deste mineral com o incremento do IMC e da idade $(\mathrm{p}<0,01)$.

Ainda na população espanhola, Rodriguéz et al. ${ }^{19}$ investigaram universitárias (20-35 anos) e identificaram maior peso corporal, IMC e circunferência da cintura entre aquelas com baixa ingestão de vitamina $\mathrm{D}$ e níveis séricos deste nutriente inferiores a $90 \mathrm{nmol} / \mathrm{L}[25(\mathrm{OH}) \mathrm{D}]$. Outros estudos corroboram estes achados e denotam ainda possível associação de outros micronutrientes com adiposidade abdominal $^{20}$ e obesidade ${ }^{21}$.

\section{Micronutrientes e estado nutricional: bases para relação}

Considerando o exposto serão descritos a seguir alguns micronutrientes e sua 
possível relação com o excesso de peso. Serão contempladas as vitaminas A, C e D, e os minerais cálcio e zinco, cujas principais fontes e recomendações encontram-se descritas no Quadro 1.

\section{Vitamina A}

A vitamina A é uma vitamina lipossolúvel, necessária para a manutenção e funcionamento dos tecidos corporais, especialmente para o crescimento e a proliferação de células epiteliais. No Brasil, a hipovitaminose A é considerada um problema de saúde pública, com índices que vão de 15,5 a 32,4\% entre crianças em idade pré-escolar ${ }^{24}$.

Um estudo realizado com $154 \mathrm{mu}$ lheres atendidas no Ambulatório de Saúde da Mulher Climatérica (ASMUC) da Universidade de São Paulo identificou $82,8 \%$ das participantes com consumo insuficiente de vitamina $\mathrm{A}$ associado à alta frequência de excesso de peso, $75 \%{ }^{23}$. De igual modo, Chaves et al. ${ }^{24}$ observaram inadequação dos níveis séricos de retinol e $\beta$-caroteno em 11,3\% e 41,7\% de indivíduos de ambos os sexos com obesidade classe III. Esses autores constataram ainda maior inadequação do nutriente entre os obesos com resistência à insulina $(\mathrm{p}<0,001)$.

Achados similares foram verificados por Barretero et al. ${ }^{25}$ em 80 pacientes com obesidade mórbida. Adicionalmente, houve correlação inversa entre o retinol sérico e o $\alpha$-tocoferol com o índice de massa corporal $(\mathrm{r}=-0,334 ; \mathrm{p}=0,002$ e $\mathrm{r}=-0,299 ; \mathrm{p}=0,007)$, respectivamente.

A possível relação da ingestão de vitamina A com excesso de peso pode estar

Quadro 1 - Nutrientes associados ao excesso de peso - principais fontes e recomendações. Chart 1 - Nutrients associated with excess weight - major sources and recommendations.

\begin{tabular}{|c|c|c|c|c|c|c|c|c|c|c|c|c|c|}
\hline \multirow[t]{3}{*}{ Nutrientes } & \multirow[t]{3}{*}{ Fontes } & \multicolumn{12}{|c|}{ Faixa Etária } \\
\hline & & \multicolumn{6}{|c|}{ Homens } & \multicolumn{6}{|c|}{ Mulheres } \\
\hline & & 9 a 13 & 14 a 18 & 19 a 30 & 31 a 50 & 51 a 70 & $>70$ & 9 a 13 & 14 a 18 & 19 a 30 & 31 a 50 & 51 a 70 & $>70$ \\
\hline $\begin{array}{l}\text { Vitamina A } \\
\text { RDA }(\mu \mathrm{g})\end{array}$ & $\begin{array}{l}\text { Fígado } \\
\text { Cenoura } \\
\text { Espinafre } \\
\text { Ovos } \\
\text { Brócolis } \\
\text { Manteiga }\end{array}$ & 600 & 900 & 900 & 900 & 900 & 900 & 700 & 700 & 700 & 700 & 700 & 700 \\
\hline $\begin{array}{l}\text { Vitamina C } \\
\text { RDA (mg) }\end{array}$ & $\begin{array}{l}\text { Acerola } \\
\text { Laranja } \\
\text { Morango } \\
\text { Alho } \\
\text { Limão }\end{array}$ & 45 & 75 & 90 & 90 & 90 & 90 & 45 & 65 & 75 & 75 & 75 & 75 \\
\hline $\begin{array}{l}\text { Vitamina E } \\
\text { RDA (mg) }\end{array}$ & $\begin{array}{l}\text { Nozes } \\
\text { Grãos integrais } \\
\text { Azeite de oliva } \\
\text { Peixes } \\
\text { Óleos vegetais }\end{array}$ & 11 & 15 & 15 & 15 & 15 & 15 & 11 & 15 & 15 & 15 & 15 & 15 \\
\hline $\begin{array}{l}\text { Vitamina D } \\
\text { RDA }(\mu \mathrm{g})\end{array}$ & $\begin{array}{l}\text { Ovos } \\
\text { Carnes } \\
\text { Leite } \\
\text { Manteiga } \\
\text { Peixes }\end{array}$ & 15 & 15 & 15 & 15 & 15 & 20 & 15 & 15 & 15 & 15 & 15 & 20 \\
\hline $\begin{array}{l}\text { Cálcio } \\
\text { RDA (g) }\end{array}$ & $\begin{array}{l}\text { Leites } \\
\text { Queijos } \\
\text { Aveia } \\
\text { Couve } \\
\text { Beterraba }\end{array}$ & 1,3 & 1,3 & 1,0 & 1,0 & 1,0 & 1,2 & 1,3 & 1,3 & 1,0 & 1,0 & 1,2 & 1,2 \\
\hline $\begin{array}{l}\text { Zinco } \\
\text { RDA (mg) }\end{array}$ & $\begin{array}{l}\text { Carnes } \\
\text { Feijão } \\
\text { Castanhas } \\
\text { Ervilha } \\
\text { Grãos integrais }\end{array}$ & 8 & 11 & 11 & 11 & 11 & 11 & 8 & 9 & 8 & 8 & 8 & 8 \\
\hline
\end{tabular}


relacionada ao metabolismo da tireóide. De acordo com Zimmermann et al. ${ }^{26}$, a deficiência deste micronutriente tem múltiplos efeitos sobre o eixo hipófise-tireóide, em virtude da modulação do metabolismo da glândula tireóide, do metabolismo periférico do hormônio tireoidiano e da produção de tirotropina (TSH) pela hipófise. Em crianças com deficiência de vitamina $\mathrm{A}$, as maiores concentrações de TSH em face da maior circulação de tiroxina total sugerem resistência central para a supressão do TSH normal pelo hormônio tireoideano ${ }^{27}$.

Além destes achados, outros estudos sugerem que a enzima retinol saturase inibe a adipogênese. Essa enzima é regulada pela transcrição dos receptores proliferadores ativados de peroxissomas (PPAR), que desempenham papel importante na biologia dos adipócitos ${ }^{28}$. No tecido adiposo, a enzima retinol saturase é expressa nos adipócitos, mas é diminuída na obesidade, possivelmente devido à maior infiltração de macrófagos, que inibe a expressão desta.

Ademais, estudo com 76 homens e mulheres com obesidade identificou associação significante entre a proteína do soro do retinol (RBP) e a obesidade e a resistência à insulina ${ }^{29}$. Notou-se que as concentrações séricas de apo-RBP foram duas vezes maiores entre os obesos $(0,90 \pm 0,62 \mathrm{mM} v s 0,44 \pm 0,56$ $\mathrm{mM}$ quando comparados aos não obesos; $\mathrm{p}$ $<0,001)$. Em contraste, a taxa de retinol/RBP foi significativamente menor entre os obesos $(0,73 \pm 0,13$ vs $0,90 \pm 0,22 ; \mathrm{p}<0,001)$. Nesses, a RBP foi associada à concentração de insulina $(r=0,26 ; p<0,05)$, índice HOMA-IR $(r=0,29$; $\mathrm{p}<0,05)$ e índice de sensibilidade à insulina $(\mathrm{r}$ $=-0,27 ; p<0,05)$. A RBP foi associada com o IMC apenas quando indivíduos obesos e não obesos foram agrupados $(r=0,25 ; p<0,01)$.

\section{Vitamina C}

A vitamina $\mathrm{C}$ ou ácido ascórbico é um micronutriente essencial que está envolvido na síntese de colágeno e nos mecanismos de defesa antioxidante. É um dos nutrientes antioxidantes mais abundantes no fluido extracelular do pulmão, estando relacionado com o sistema imunológico ${ }^{22}$.

No estudo Brazos $^{10}$, a ingestão de vitamina $\mathrm{C}$ foi adequada em apenas $20 \%$ da população estudada, e em outro estudo realizado com adultos do município do Rio de Janeiro que apresentavam síndrome metabólica, o consumo de vitamina $\mathrm{C}$ foi insuficiente em 93\% da população ${ }^{30}$.

Um estudo transversal de base populacional realizado com 926 mulheres (40-60 anos) avaliou a ingestão alimentar por meio de um questionário semiquantitativo de frequência alimentar e identificou associação da obesidade central com baixo nível de atividade física (odds ratio: 2,11; IC 95\%: 1.40 - 2.53), tabagismo $(1,21 ; 1,02-$ $1,56)$, menopausa $(1,22 ; 1,02-1,61)$, baixa ingestão de vitamina $C(2,31 ; 1,25-4,25) \mathrm{e}$ de cálcio $(1,30 ; 1,07-3,78)^{31}$.

Canoy et al..$^{20}$ encontraram resultados semelhantes em uma população européia ( $n=19.068)$, com idade entre 45-79 anos. As baixas concentrações plasmáticas de ácido ascórbico foram inversamente relacionadas com aumento nos quartis da razão cintura-quadril ( $p<0,001)$ e no índice de massa corporal em mulheres $(p<0,001)$.

Além da possível associação com o excesso de peso, estudo prospectivo europeu, com duração de 12 anos, verificou associação inversa entre a concentração sérica de vitamina $\mathrm{C}$ e o risco de diabetes mellitus tipo $2^{32}$. Ademais, Holt et al. ${ }^{33}$ identificaram correlação inversa do consumo de vitamina $\mathrm{Ce}$ a concentração de proteína C reativa (PCR), um marcador de inflamação $(\mathrm{r}=-0,13 ; \mathrm{p}=$ 0,03 ) entre 285 alunos (13-17 anos) de uma escola pública de Minessota. Fredrikson et al. ${ }^{34}$ também observaram correlação inversa da ingestão do referido nutriente com os níveis de PCR e IMC.

Essas associações podem estar relacionadas ao fato de o nutriente participar da síntese de catecolaminas (convertendo dopamina em noradrenalina) e atuar como um cofator na síntese de carnitina, envolvida na oxidação da gordura e intervindo no transporte de ácidos graxos no interior da mitocôndria, fundamental para produção de energia ${ }^{33,34}$. Nota-se que os níveis do 
aminoácido estão diretamente relacionados às concentrações séricas de vitamina $\mathrm{C}, \mathrm{o}$ que pode contribuir, quando deficientes, para incremento da adipogênese e favorecimento das condições a ela associadas, como obesidade e diabetes ${ }^{35}$.

\section{Vitamina D}

A vitamina D é um hormônio esteróide essencial para a homeostase do cálcio e manutenção da saúde óssea. É sintetizada naturalmente pela pele após exposição à radiação ultravioleta do sol e tem a 25-diidroxi-vitamina D - 25(OH)D - como seu metabólito mais abundante na circulação e indicador do estado nutricional dessa vitamina ${ }^{22}$.

Dados de 46 estudos recentes da Europa, América do Norte, do Sudeste Asiático e a região do Pacífico Sul, indicam que um baixo status da vitamina D e cálcio é altamente prevalente na população geral $(30-80 \%)$, atingindo ambos os $\operatorname{sexos}^{36}$. A prevalência desta ingestão insuficiente é mais elevada na população idosa, crianças e mulheres jovens em idade fértil, minorias étnicas e imigrantes, bem como pessoas de nível socioeconômico mais baixo ${ }^{36}$.

Forrest et al. ${ }^{37}$ avaliaram a prevalência da deficiência de vitamina $\mathrm{D}$ e seus correlatos em subgrupos da população americana, através de dados de 4.495 participantes da National Health and Nutrition Examination Survey - 2005-2006 (NHANES). A prevalência global de deficiência de vitamina D - concentrações de 25(OH)D inferiores a $20 \mathrm{ng} / \mathrm{mL}$ - foi de 41,6\% (IC95\%: 36,6\% $46,8 \%$ ), sendo superior em negros (82,1\%) e hispânicos (69,2\%). Além disso, a deficiência foi mais presente entre participantes com baixa escolaridade, obesidade e hipercolesterolemia $(\mathrm{p}<0,001)$.

Outras avaliações de dados do NHANES ${ }^{18}$ demonstraram que a alta concentração de $25(\mathrm{OH}) \mathrm{D}$ esteve associada a uma melhor qualidade da dieta, menor percentual de gordura corporal e menor frequência de distúrbios metabólicos $(\mathrm{p}<0,005)$.

No Brasil, Peters et al..$^{38}$ avaliaram 136 adolescentes de uma região rural de São
Paulo e identificaram que apenas 14,9\% apresentavam ingestão adequada de vitamina D (3,5; 3,0 - 3,9mg/dia), sendo a insuficiência bioquímica deste nutriente observada em $60 \%$ dos participantes. No estudo Brazos, 99\% da população estudada apresentou inadequação no consumo de vitamina $\mathrm{D}^{10}$.

Estudos demonstram que adultos obesos e crianças têm baixas concentrações séricas de $25(\mathrm{OH}) \mathrm{D}$ e elevado nível hormônio da paratireóide (PTH) ${ }^{39,40}$. Ademais, indivíduos adultos com hipovitaminose $\mathrm{D}$ parecem apresentar maior risco de resistência à insulina e síndrome metabólica, em virtude de disfunção das células beta pancreáticas e maior ocorrência de diabetes mellitus tipo $2^{41}$.

Acredita-se que a insuficiência de vitamina D não seja consequência da menor exposição solar, mas um dos fatores que desencadeia o acúmulo de gordura corporal ${ }^{40}$. Esse processo pode estar ligado ao depósito de vitamina $\mathrm{D}$ nos adipócitos, diminuindo a sua biodisponibilidade e provocando uma cascata de reações pelo hipotálamo que resulta no aumento da sensação de fome e diminuição do gasto energético ${ }^{40}$. Tal situação também gera aumento nos níveis de paratormônio (PTH), diminuição da sensibilidade à insulina e aumento desproporcional na concentração de cálcio intracelular.

Há evidências de estudos epidemiológicos com a vitamina $\mathrm{D}$, ensaios clínicos de intervenção, bem como estudos com modelos animais de doenças humanas que o comprometimento no status da vitamina $\mathrm{D}$ e do cálcio advindos da alimentação inadequada é um fator predisponente para um grande número de patologias, incluindo vários tipos de câncer, doenças infecciosas, inflamatórias e autoimunes, cardiovasculares e metabólicas, como obesidade e hipertensão ${ }^{37}$.

\section{Cálcio}

O cálcio, mineral mais comum no organismo humano, é essencial para a mineralização de ossos e dentes; é cofator da cascata de coagulação sanguínea; atua na 
transmissão nervosa, contração muscular e também na liberação da insulina ${ }^{22}$. Além disso, para alguns pesquisadores, esse nutriente atua na cinética lipídica adipocitária, sendo que o seu aumento intra-adipócito altera o balanço entre a síntese e a utilização de lipídios, favorecendo a lipogênese. Estima-se que os níveis de ingestão de cálcio possam explicar as variações de $3 \%$ a $10 \%$ do peso corporal de um indivíduo adulto ${ }^{22}$.

Santos et al. ${ }^{42}$ avaliaram 96 adolescentes pós púberes, com idade média de 16,6 \pm 1,3 anos e detectaram menor consumo de cálcio entre aqueles com obesidade $(585,2$ $\pm 249,9$ vs $692,1 \pm 199,5 \mathrm{mg}$ ). A ingestão de cálcio foi inversamente associada com a gordura corporal e a resistência à insulina, principalmente entre as adolescentes com excesso de peso.

Em adultos australianos, Soares et al. ${ }^{43}$ verificaram contribuição da ingestão de cálcio tanto para o IMC quanto para circunferência da cintura em homens e mulheres. Os resultados demonstraram que a ingestão do mineral apresentava relação inversa com a adiposidade abdominal. Loss et al. ${ }^{44}$ observaram resultados semelhantes.

A ingestão de cálcio possivelmente se encontra relacionada ao controle do peso por diversos mecanismos. No trato intestinal, proporciona uma ligação com os ácidos graxos, limitando a sua absorção. A participação do mineral na regulação da temperatura corporal e no aumento da termogênese também tem sido apontada como efeito antiobesidade. A concentração de cálcio intracelular exerce função importante em vários processos metabólicos, influenciando a concentração de hormônios como o paratormônio e a vitamina D. A baixa ingestão deste mineral proporciona aumento dos níveis séricos de vitamina $\mathrm{D}$ e paratormônio, promovendo o influxo de cálcio no adipócito e consequente aumento da lipogênese e redução da lipólise ${ }^{45}$.

\section{Zinco}

O zinco é um mineral essencial que está envolvido com o crescimento, o sistema imunológico e a cognição ${ }^{22}$. Suas funções têm sido agrupadas em estrutural - dando suporte para manutenção da forma e disposição espacial das enzimas e de proteínas, função enzimática -, manutenção do balanço ácidobásico do sangue e atividades catalíticas de enzimas, e função reguladora - atuação na atividade dos neurônios e na memória $^{22}$.

A deficiência de zinco moderada e grave tem sido cada vez mais detectada, principalmente nos países em desenvolvimento, onde estudos bem delineados têm mostrado a sua importância clínica, em que se observa retardo no crescimento, diarréia, pneumonia, malária e desenvolvimento cerebral prejudicado ${ }^{46}$. Uma revisão de literatura realizada por Cozzolino ${ }^{47}$ denotou maior prevalência de insuficiência de zinco entre crianças com obesidade ( $48 \%$ vs $9,5 \%$ entre as crianças eutróficas).

Sánchez et al. ${ }^{18}$ avaliaram uma população adulta do sul da Espanha e verificaram inadequação de zinco e ferro em 56,45\% e $22,45 \%$ da amostra, respectivamente. As concentrações plasmáticas de zinco apresentavam-se inferiores aos valores de referência em $17,8 \%$ da população e foram relacionadas de maneira inversa com idade e IMC $(\mathrm{p}<0,001)$. Achados semelhantes foram descritos por Marreiro et al. ${ }^{48} \operatorname{com} 44$ crianças e adolescentes.

Há evidências de que a participação do zinco estimula a atividade do receptor de insulina tirosina quinase, que, posteriormente, por meio do estímulo pós-receptor, parece aumentar a translocação dos transportadores de glicose dos seus sítios intracelulares para a membrana plasmática. Associado a esse fato, muitos estudos têm demonstrado que as concentrações de zinco no plasma, nos eritrócitos e no soro de indivíduos obesos estão diminuídas, e que a suplementação com esse mineral reduz a resistência à insulina ${ }^{49}$.

Adicionalmente, sugere-se uma interrelação do mineral com a concentração de leptina, hormônio relacionado à saciedade. Em indivíduos com níveis inadequados de zinco, a concentração de leptina é reduzida. 
Quadro 2 - Evidências de associação dos micronutrientes com o excesso de peso: possíveis mecanismos.

Chart 2 - Evidence of association of micronutrients with excess weight: possible mechanisms.

\begin{tabular}{|l|l|}
\hline Nutrientes & Mecanismos \\
\hline Vitamina A & Participação no funcionamento da tireóide \\
Vitamina C & Síntese de carnitina e processo de oxidação de gordura \\
Vitamina D & Saciedade, redução do gasto energético e aumento do paratormônio-PTH \\
Cálcio & Termogênese e processo lipogênese/lipólise \\
Zinco & Regulação hormonal - insulina e leptina \\
\hline
\end{tabular}

A suplementação de zinco proporciona aumento na produção de interleucina 2 e fator de necrose tumoral-TNF $\alpha$, sendo estes relacionados ao aumento da produção de leptina ${ }^{50}$. Entretanto, ainda não está claro se este mineral controla a concentração de leptina direta ou indiretamente por meio do aumento dos marcadores. Os possíveis mecanismos da associação entre os micronutrientes e o excesso de peso encontram-se sintetizados no Quadro 2.

\section{Conclusões}

O incremento do excesso de peso e da obesidade na população mundial adulta é considerado atualmente um problema de saúde pública e pode se associar as transformações ocorridas no campo social, demográfico, cultural e de saúde nas últimas décadas. Essas modificações favoreceram a adoção de novos modos de vida, incluindo o consumo excessivo de alimentos industrializados e o consumo insuficiente de frutas, legumes e verduras, contribuindo para as inadequações de micronutrientes.

As evidências aqui apontadas demonstraram que muitos micronutrientes - vitaminas A, C, E e D , cálcio e zinco - estão envolvidos em processos metabólicos e endócrinos importantes no que diz respeito à gênese/controle do excesso de peso. Por este motivo, a ingestão dietética adequada torna-se fundamental como fator de proteção para ocorrência de obesidade e outras DANT.

Portanto, a promoção de hábitos alimentares saudáveis por meio de práticas educativas se faz necessária para maior conscientização da população sobre alimentação e qualidade de vida. A associação direta entre consumo de micronutrientes, hábitos saudáveis e melhor qualidade de vida denota que estes necessitam ser componentes prioritários nas estratégias de Saúde Pública, a fim de deter o avanço da obesidade e suas complicações.

\section{Referências}

1. Kac G, Velásquez-Meléndez G. A transição nutricional e a epidemiologia da obesidade na América Latina. Cad Saúde Pública 2003; 19(1): 4-52. Brasil. A Lei nº 11.346, de 15 de setembro de 2006, cria o Sistema Nacional de Segurança Alimentar e Nutricional - SISAN, com vistas a assegurar o direito humano à alimentação adequada $\mathrm{e}$ dá outras providências. Diário Oficial da União 2006; 18 set.

2. Popkin BM. The nutrition transition and obesity in the developing world. J Nutr 2001; 131: 871S-3S.
3. Instituto Brasileiro de Geografia e Estatística. Pesquisa de Orçamentos Familiares 2002-2003: Análise da disponibilidade domiciliar de alimentos e do estado nutricional no Brasil. Rio de Janeiro. IBGE; 2004.

4. Instituto Brasileiro de Geografia e Estatística. Pesquisa de Orçamentos Familiares 2008-2009: Antropometria e Estado nutricional de crianças, adolescentes e adultos no Brasil. Rio de Janeiro. IBGE; 2010. 
5. Brasil. Ministério da Saúde. Secretaria de Vigilância em Saúde. Secretaria de Gestão Estratégica e Participativa. Vigitel Brasil 2008: vigilância de fatores de risco e proteção para doenças crônicas por inquérito telefônico. Brasília; 2009. 39p.

6. Monteiro CA, Mondini L, Costa, RBL. Mudanças na composição e adequação nutricional da dieta familiar nas áreas metropolitanas do Brasil (1988-1996). Rev Saúde Pública 2000; 34(3): 251-8.

7. World Health Organization. Diet, nutrition and prevention of chronic diseases: report of a joint WHO/FAO expert consultation. Geneva; 2002.11p.

8. Instituto Brasileiro de Geografia e Estatística - IBGE. Pesquisa de orçamentos familiares 2002-2003: primeiros resultados: Brasil e grandes regiões. Rio de Janeiro: IBGE, Coordenação de Índices de Preços 2004. 276 p.

9. World Health Report 2001: Reducing risks, promoting healthy life. Geneva: World Health Organization; 2001. p. 7-9.

10. Pinheiro MM, Schuch NJ, Genaro PS, Ciconelli RM, Ferraz MB, Martini LA. Nutrient intakes related to osteoporotic fractures in men and women: The Brazilian Osteoporosis Study (BRAZOS). Nutr J 2009: 1-8.

11. Majem SL et al. Trends in energy and nutrient intake and risk of inadequate intakes in Catalonia, Spain (19922003). Public Health Nutrition 2007; 10(11A), 1354-67.

12. World Health Organization. The world health report 2002: reducing risks, promoting healthy life. Geneva; 2002.

13. Azadbakht L, Esmaillzadeh A. Dietary and non-dietary determinants of central adiposity among Tehrani women. Public Health Nutrition 2007; 1(5): 528-34.

14. Lopes ACS, Caiaffa WT, Sichieri R, Mingoti SA, LimaCosta MF. Consumo de nutrientes em adultos e idosos em estudo de base populacional: Projeto Bambuí. Cad Saúde Pública 2005; 21(4): 1201-9.

15. Velásquez-Meléndez G, Martins IS, Cervato AM, Fornés NS, Marucci MFN. Consumo alimentar de vitaminas e minerais em adultos residentes em área metropolitana de São Paulo, Brasil. Rev Saúde Pública 1997; (31): 15762.

16. Abreu WC, Franceschini SCC, Tinoco ALA, Pereira CAS, Silva MMS. Inadequação no consumo alimentar e fatores interferentes na ingestão energética de idosos matriculados no programa municipal da terceira idade de Viçosa (MG). Rev Baiana de Saúde Pública maio/agos 2008; 32(2): 190-202.

17. Suliburska J et al. Dietary Intake and Serum and Hair Concentrations of Minerals and their Relationship with Serum Lipids and Glucose Levels in Hypertensive and Obese Patients with Insulin Resistance. Biol Trace Elem Res 2011; 139: 137-50.
18. Sánchez C, Jurado LM, Planells E, Llopis J, Aranda P. Assessment of iron and zinc intake and related biochemical parameters in an adult Mediterranean population from southern Spain: influence of lifestyle factor. J Nutr Biochem 2009; 20: 125-31.

19. Rodriguéz RE et al. Vitamin D in Overweight/Obese Women and Its Relationship With Dietetic and Anthropometric Variables. Obesity 2009; 17: 778-82.

20. Canoy D et al. Plasma ascorbic acid concentrations and fat distribution in 19.068 British men and women in the European Prospective Investigation into Cancer and Nutrition Norfolk cohort study. Am J ClinNutr 2005; 82: 1203-9.

21. Lagunova Z et al. Serum 25-Hydroxyvitamin D Is a Predictor of Serum 1.25-Dihydroxyvitamin D in Overweight and Obese Patients. J Nutr 2011; 141: 112-7.

22. Fisberg $\mathrm{M}$ et al. O papel dos nutrientes no crescimento e desenvolvimento infantil.São Paulo: SARVIER; 2008. $186 \mathrm{p}$.

23. Montilla RNG, Marucci MFN, Aldrighi JM. Avaliação do estado nutricional e do consumo alimentar de mulheres no climatério. Rev Assoc Med Bras 2003; 49(1): 91-5.

24. Chaves GV et al. Non-alcoholic Fatty Liver Disease and Its Relationship with the Nutritional Status of Vitamin A in Individuals with Class III Obesity. Obes Surg 2008; 18 : 378-85.

25. Barretero JIB et al. Retinol and -Tocopherol in Morbid Obesity and Nonalcoholic Fatty Liver Disease. Obes Surg 2010; 20: 69-76.

26. Zimmermann MB. Interactions of vitamin A and iodine deficiencies: effects on the pituitary-thyroid axis. Int J Vitam Nutr Res 2007; 77(3): 236-40.

27. Schupp $\mathrm{M}$ et al. ProcRetinol saturase promotes adipogenesis and is downregulated in obesity. Natl Acad Sci USA 2009; 106(4): 1105-10.

28. Mills JP; Furr HC; Tanumihardjo AS. Retinol to retinolbinding protein (RBP) is low in obese adults due to elevated apo-RBP. Exp Biol Med 2008; 233(10): 1255-61.

29. Fernandes M, Paes C, Nogueira C, Souza G, Aquino L, Borges F et al. Perfil de consumo de nutrientes antioxidantes em pacientes com síndromemetabólica/ Profile of antioxidant nutrient intake in patients with metabolic syndrome. Rev Ciênc Médica 2007; 16(4/6): 209-19.

30. Ramalho RA, Braun F, Gomes AIS, Taveira M, Souza LB, Ribeiro BG. Lipid profile and consumption of dietary antioxidants in overweight and obese individuals, users of the health promotion center - Petrobrás, Rio de Janeiro, Brazil. Clin Nutr 2004; 23(4): 1005-6.

31. NeumannAICP,ShirassuMM, FisbergRM.Consumo de alimentos de risco e proteção para doenças cardiovasculares entre funcionários públicos. Rev Nutr 2006; 19(1): 19-28. 
32. Harding A, Wareham NJ, Bingham SA, Khaw K, Luben R, Welch A et al. Plasma vitamin C level, fruit and vegetable consumption, and the risk of new-onset type 2 diabetes mellitus. The European Prospective Investigation of Cancer-Norfolk Prospective Study. Arch Intern Med 2008; 168(14): 1493-9.

33. Holt EM, Steffen LM, Moran A, Basu S, Steinberger J, Ross JA et al. Fruit and vegetable consumption and its relation to markers of inflammation and oxidative stress in adolescents. J Am Diet Assoc 2009; 109(3): 414-21.

34. Fredrikson GN et al. Association Between Diet, Lifestyle, Metabolic Cardiovascular Risk Factors and Plasma C Reactive Protein Levels. Metabolism 2004; 53(11): 143642.

35. Jonsthon CS et al. Plasma Vitamin C Is Inversely Related to Body Mass Index and Waist Circumference but Not to Plasma Adiponectin in Nonsmoking Adults. J Nutr Washington 2007; 137(7): 1757-62.

36. Su X, Zemel MB. 1alpha, 25-dihydroxyvitamin D and corticosteroid regulate adipocyte nuclear vitamin D receptor. Int J Obes (Lond) 2008; 32(8): 1305-11.

37. Forrest KYZ, Stuhldreher WL. Prevalence and correlates of vitamin D deficiency in US adults. Nutr Res 2011; 31: 48-54.

38. Peters BSE, dos Santos LC, Fisberg M, Wood RJ, Martini LA. Prevalence of Vitamin D Insufficiency in Brazilian Adolescents. Ann Nutr Metab 2009; 54: 15-21.

39. Peterlik M, Boonen S, Cross HS, Lamberg-AllardtC. Vitamin D and Calcium Insufficiency-Related Chronic Diseases: an Emerging World-Wide Public Health Problem. Int J Environ Res Public Health 2009; 6(10): 2585-607.

40. Schuch NJ, Garcia VC, Martini LA. Vitamina D e doenças endocrinometabólicas. Arq Bras Endocrinol Metab 2009; 53(5): 625-33.

41. Snijder MB, van Dam RM, Visser M, Deeg DJH, Dekker JM, Bouter LM et al. Adiposity in relation to vitamin D status and parathyroid hormone levels: a populationbased study in older men and women. J Clin Endocrinol Metab 2005; 90: 4119-23.
42. Santos LC, Cintra IP, Fisberg M, Martini LA. Ingestão de cálcio e indicadores antropométricos entre adolescentes. Rev Nutr Campinas 2007; 20(3): 275-83.

43. Soares MJ, Binns C, Lester L. Higher intakes of calcium are associated with lower BMI and waist circumference in Australian adults: an examination of the 1995 National Nutrition Survey. Asia Pac Clin Nutr 2004; 13: 585.

44. Loos RJ, RankinenT, Leon AS, Skinner JS, Wilmore JH, Rao DC, Bouchard C. Calcium intake is associated with adiposity in Black and White woman of the Heritage Family Study. J Nutr 2004; 134(7): 1772-8.

45. Zemel MB, Thompson W, Milstead A, Morris K, Campbell $P$. Calcium and dairy acceleration of weight and fat loss during energy restriction in obese adults. Obes Res 2004; 12(4): 582-90.

46. Hambidge M. Human zinc deficiency. J Nutr 2000; 130S: 1344-9.

47. Cozzolino SMF. Deficiências de minerais. Estudos Avançados 2007; 21(60): 119-26.

48. Marreiro DN, Fisberg M, Cozzolino SMF. Zinc Nutritional Status and Its Relationship with Hyperinsulinemia in Obese Children and Adolescents. Biol Trace Elem Res 2004; 100: 137-49.

49. Marreiro DN, Fisberg M, Cozzolino SMF. Zinc nutritional status in obese children and adolescents. Biol Trace Elem Res 2002; 86(2):107-22.

50. Marreiro DN. Efeito da suplementação com zinco na resistência à insulina em mulheres obesas [tese de doutorado].São Paulo: Faculdade de Ciências Farmacêuticas - USP; 2002.

Recebido em: 22/03/11 Versão final apresentada em: 01/10/11 Aprovado em: 25/11/11 


\section{Errata Correction \\ Consumo de micronutrientes e excesso de peso: existe relação? \\ Micronutrient consumption and overweight: Is there a relationship?}

\section{Volume 15, número 1, março/2012, Página 89:}

Onde se lê:

Quadro 1 - Nutrientes associados ao excesso de peso - principais fontes e recomendações. Chart 1 - Nutrients associated with excess weight - major sources and recommendations.

\begin{tabular}{|c|c|c|c|c|c|c|c|c|c|c|c|c|c|}
\hline \multirow[t]{3}{*}{ Nutrientes } & \multirow{3}{*}{ Fontes } & \multicolumn{12}{|c|}{ Faixa Etária } \\
\hline & & \multicolumn{6}{|c|}{ Homens } & \multicolumn{6}{|c|}{ Mulheres } \\
\hline & & 9 a 13 & 14 a 18 & 19 a 30 & 31 a 50 & 51 a 70 & $>70$ & 9 a 13 & 14 a 18 & 19 a 30 & 31 a 50 & 51 a 70 & $>70$ \\
\hline $\begin{array}{l}\text { Vitamina D } \\
\text { RDA }(\mu \mathrm{g})\end{array}$ & $\begin{array}{l}\text { Ovos } \\
\text { Carnes } \\
\text { Leite } \\
\text { Manteiga } \\
\text { Peixes }\end{array}$ & 600 & 600 & 600 & 600 & 600 & 800 & 600 & 600 & 600 & 600 & 600 & 800 \\
\hline
\end{tabular}

O correto é:

Quadro 1 - Nutrientes associados ao excesso de peso - principais fontes e recomendações. Chart 1 - Nutrients associated with excess weight - major sources and recommendations.

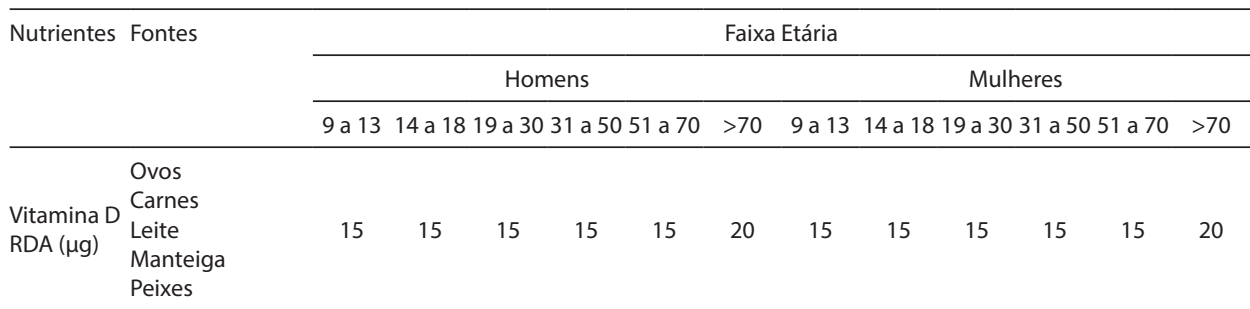

